\title{
LONG-LIVED PLASMA CELLS SECERET ANTIBODIES POSSESSING HIGH AFFINITY IN CHANNEL CATFISH
}

\author{
Liting Wu, Shengli Fu, Xiaoxue Yin, Zheng Guo, Anli Wang, Jianmin Ye $§$
}

\begin{abstract}
Guangdong Provincial Key Laboratory for Healthy and Safe Aquaculture, Guangdong Engineering Research Center for Environmentally-friendly Aquaculture, College of Life Science, South China Normal University, Guangzhou 510631, People's Republic of China
\end{abstract}

\begin{abstract}
Recent mammalian studies have shown that long-lived plasma cells (LLPCs) provide persistent specific antibody titer resulting in humoral immunological memory, which constitute a new kind of "memory-providing cells". The LLPCs have been identified in rainbow trout, which provide similar constant expression of specific antibodies over a period of 35 weeks. However, there are little studies on whether the challenge to become as a LLPC in teleost is driven by affinity selection. In this study, channel catfish was immunized with TNP-KLH to examine the affinity of antibodies secreted by the LLPCs, the affinity of serum antibodies over response, the generation, tissue distribution, and the movement of LLPCs. TNP-specific LLPCs began to be generated at 4 weeks post-immunization, achieved a maximal number by week 8 of the response, and maintained a comparable level throughout the test period of 18 weeks, which is correlated to the dynamics of serum antibody affinity maturation in the response. LLPCs appear to mostly reside within, or migrate to the anterior kidney, and are located in spleen with a small portion. The antibody affinity produced by the anterior kidney LLPCs was shown to be much higher than that of other ASCs, which may indicate affinity-driven selection appears to play a significant role in plasma cell fate. Upon resolving the affinity-dependent mechanism of LLPCs, some important implications will be proposed for the development of immunological memory in teleost and the design of efficient aquaculture vaccine.
\end{abstract}

\section{KEYWORDS}

Long-lived plasma cells, antibody, affinity, affinity-driven selection, immunological memory

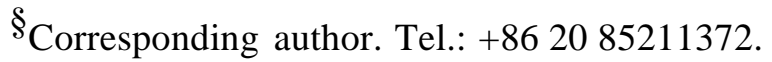

E-mail address: yjmying@126.com 